\title{
Tectal pathways of regenerating goldfish optic axons after nasal or temporal half retinal removal
}

\author{
MARTIN F. HUMPHREY ${ }^{1, *}$ and CLAUDIA A. O. STUERMER ${ }^{2}$ \\ ${ }^{1}$ Max-Planck-Institut für Hirnforschung, Frankfurt/M, FRG \\ ${ }^{2}$ Friedrich-Miescher-Laboratorium der Max-Planck-Gesellschaft, Tübingen, FRG \\ ${ }^{*}$ Present address for correspondence: Miami Project to Cure Paralysis, 1600 N.W. 10th Avenue, R-48, Miami, Florida 33136, USA
}

\section{Summary}

The tectal pathways of regenerating goldfish optic axons are abnormal but not random. The relative proportion of temporal axons is highest in rostral tectum (65\%) drops in midtectum $(31 \%)$ and is very low in caudal tectum ( $4 \%$ ). By contrast, nasal axons proceed into caudal tectum and are therefore relatively evenly distributed throughout the tectum. In this study, we have tested whether temporal axons are confined to rostral tectum by the presence of nasal axons in caudal tectum or whether they have a preference for rostral tectum regardless of other axons. We similarly tested whether nasal axons would grow preferentially into caudal tectum in the absence of temporal axons.

At the time of optic nerve section either the nasal or temporal half retina was removed. Either 35 or 70 days after nerve section, the regenerating optic axons were labelled with HRP and both their pathways and distribution determined in DAB-reacted tectal wholemounts.
In the absence of nasal axons, the relative density of temporal axons in rostral, mid and caudal tectum was $70 \%, 28 \%$ and $2 \%$, respectively. The corresponding values for nasal axons, in the absence of temporal axons, were $30 \%, 40 \%$ and $30 \%$, respectively. Thus, the overall distribution of nasal and temporal axons in the half retinal regenerates was similar to that of whole retinal regenerates, demonstrating that the retinotopic preferences of the axons were not dependent upon interaxonal interactions.

Thus, nasal and temporal axons obviously discriminate between rostral and caudal tectum despite pathway disorganization and the absence of axons from the opposite hemiretina. This is consistent with axonal growth being under the influence of positional markers in tectum.

Key words: retinotectal, topographic projections, positional information, axonal regeneration.

\section{Introduction}

The pathways laid down by axons in locating their target region provide clues about the growth strategy used for locating that region (Fujisawa et al. 1982). Recent anatomical studies in goldfish, in which the pathways of retinal axons to their target sites in the optic tectum have been investigated, have provided new information concerning the pathway organization of both normal axons (Cook \& Rankin, 1984; Easter \& Stuermer, 1984; Stuermer \& Easter, 1984a) and axons regenerating after optic nerve section (Rankin \& Cook, 1986; Stuermer, 1986; Stuermer \& Easter, 1984b). In light of these findings, the view conveyed by Attardi \& Sperry (1963) that regenerating axons follow normal routes to their targets has had to be modified.

It has been shown that regenerating axons do not recapitulate the spatiotemporal order of their normal predecessors but course through abnormal routes and inappropriate tectal territories (Stuermer, 1986, $1988 a, b)$. It may therefore be possible that regenerating axons locate their appropriate termination region largely by a trial and error mechanism rather than by following positional information on the tectum.

Recent quantitative evaluations of the tectal distribution of axons from known retinal regions have 
revealed, however, that the vast majority of temporal axons course through the appropriate rostral tectal half (Stuermer, 1987a,b). Thus, their relative density declined from $65 \%$ in rostral tectum to $31 \%$ in midtectal regions and $4 \%$ in caudal tectum. Nasal axons, on the other hand, were distributed at a similar density in rostral and midtectal regions and only declined to $15 \%$ caudal to their appropriate termination region. Furthermore, axons misrouted into inappropriate tectal territories were observed to undergo course corrections directed towards their target sites (Stuermer, 1986). Thus, regenerating axons exhibited a clear preference for their correct tectal territories, despite their abnormal pathways.

The degree to which the above pattern of ingrowth is a consequence of interactions between the ingrowing axons themselves, as opposed to interactions between the axons and positional cues in the tectum, is unknown. During the early period of axonal ingrowth, the axons are closely associated with one another and so there is plenty of opportunity for them to interact (Murray, 1976; Murray \& Edwards, 1982; Stuermer \& Easter, 1984b). It has also been shown that axons can exclude or displace one another from regions within the target (Meyer, 1979; Sharma \& Tung, 1979). Thus it is possible that axons appropriate to a given tectal region prevent excessive invasion by inappropriate axons. The presence of axons appropriate to a region may also be a stimulus for withdrawal of inappropriate branches (Fujisawa, 1981; Meyer, 1980; Schmidt \& Edwards, 1983; Stuermer, $1987 a, b)$.

Attardi \& Sperry (1963), using silver staining, provided anatomical evidence that both nasal and temporal axons grew selectively to their appropriate tectal halves even in the absence of input from the opposite half of the retina. However, more recent HRP studies (Rankin \& Cook, 1986; Stuermer, 1986; Stuermer \& Easter, 1984) have shown that their description of the axonal pathways was inaccurate and understaining may have been a problem. More modern anatomical techniques have produced a similar overall picture but none were suitable for detailed axonal tracing (Meyer, 1980; Stuermer, 1981; Meyer et al. 1985). Recent electrophysiological studies even suggest that nasal axonal arbours are present over the entire tectum as early as $46-60$ days after nerve crush following temporal half retinal removal (Meyer, 1987).

Therefore, we have reinvestigated the distribution, pathways and branching patterns of regenerating nasal and temporal axons following optic nerve section and removal of their respective opposite retinal half. By using anterograde transport of HRP to label the entire nerve and also discrete bundles of axons, we have been able to trace the pathways with greater accuracy than previous studies.

Our results have shown that temporal axons preferentially course over rostral tectum, even in the absence of nasal axons, and nasal axons proceed into caudal tectum even when temporal axons are absent. The pathways taken in each case closely resemble those of whole retinal regenerates (Stuermer, $1987 a, b)$. Additionally, axonal ramifications in the inappropriate tectal half did not increase in the absence of the axons appropriate to that half.

Preliminary accounts of this study have been published in abstract form (Humphrey \& Stuermer, 1987).

\section{Materials and methods}

Adult goldfish, Carassius auratus, $4-6 \mathrm{~cm}$ in length were maintained at low density in aerated aquaria at room temperature $\left(17-20^{\circ} \mathrm{C}\right)$. For all surgical procedures, the fish were anaesthetized by immersion in a $0.1 \%$ aqueous solution of tricaine methane sulphonate (MS222). A total of 42 fish was operated bilaterally for this study. Those examined had to meet strict criteria of complete half retinal removal, an undamaged remaining retinal half (assessed by whole mounting) and strong axonal labelling with low background. The number examined is indicated in each relevant section.

Optic nerves were severed within the orbit taking care not to cut the blood vessels supplying the eye. An incision was then made in the cornea, the lens was reflected from the eye and the retina cut with a razor blade along a vertical line adjacent to the optic nerve head. The half retina that was isolated from the optic nerve head was removed by gentle suction. The lens and corneal flap were then replaced. Both temporal and nasal half removals were done after nerve cut. Similar removals were done without optic nerve cut to establish the location in the tectum of the representation of the cut retinal edge. For direct comparison of half retinal regeneration with regeneration of the whole retina, some whole retinal regenerates had half of the regenerate retina removed several days prior to labelling of the optic nerve. Thus, the representation of the half retina after whole retinal regeneration could also be examined.

To label the entire optic nerve a Fibrospum (Promonta) pledget soaked in a saturated solution of HRP (horseradish peroxidase, Type VI, Miles) in 0.1 M-phosphate buffer (PB) $\mathrm{pH} 7: 4$ was applied to the distal nerve stump after severing the nerve between the eye and initial lesion site. To ensure maximal uptake, the nerve was severed with a sharp razor blade. Small groups of axons were labelled by damaging a few fascicles of axons within the retina using a glass micropipette coated at the tip with HRP crystals. Following either procedure, animals were left for 2-3 days prior to reanaesthetization, perfusion with $0.6 \%$ saline and removal of the retinae and tecta which were immediately placed into a solution of $0.05 \%$ diaminobenzidine (DAB), $0.01 \%$ hydrogen peroxide in $0.2 \mathrm{M}-\mathrm{PB} \mathrm{pH} 7.2$ for $1 \mathrm{~h}$. The tecta were then fixed for $2 \mathrm{~h}$ in $4 \%$ glutaraldehyde in $0.2 \mathrm{M}$ - 
PB $\mathrm{pH} 7.2$ prior to flattening on a gelatinized slide followed by dehydration and mounting in Permount. The retinae were fixed in $10 \%$ PB formalin $\mathrm{pH} 7.4$ for several days, then flattened and air dried onto $5 \%$ gelatinized slides, dehydrated and mounted in Permount.

The quantification methods will be explained in the relevant results sections.

\section{Results}

In order to examine the overall pattern of regeneration, the whole optic nerve was labelled in a series of both nasal $(n=11)$ and temporal $(n=6)$ half retinal regenerates, these were compared with control animals where a half retina was removed but the nerve was not sectioned at the time of removal $(n=10)$. Attention was focussed on two periods of regeneration. The first was 35-40 days after nerve section when the majority of axons should still have been growing towards their target regions and very few would have started to form terminal arborizations. The second was at 75-76 days when the tectum should already be densely innervated and mature arbours are identifiable.

\section{Distribution of axons}

In the control fish, which had not received optic nerve section, the pattern of labelling following removal of either the nasal or temporal half of the retina was as would have been expected from previous studies of the normal pathways (Stuermer \& Easter, 1984a; Easter \& Stuermer, 1984). Temporal axons were confined to the rostral tectum in both the superficial fibre layer (SO) and synaptic layer (SGFS). There was a relatively sharp boundary between labelled and unlabelled tectum located approximately halfway along the rostrocaudal extent of the tectum. Terminal arbours in the deepest optic termination layers projected slightly farther into the denervated tectum, presumably reflecting their greater size (Stuermer, 1984). Nasal axons were present in rostral tectum both within superficial fascicles and as fibres of passage (the 'extrafascicular' axons of Easter \& Stuermer, 1984a; Stuermer \& Easter, 1984a) in the synaptic layer. Terminal arbours of nasal axons were confined to the caudal tectum.

In the regenerates that had half of the retina removed at the time of nerve section (i.e. half retinal regenerates) the fibre order was very different from normal. Also, in contrast to the findings of Attardi and Sperry, temporal axons were not completely restricted to the appropriate rostal tectum as some had also invaded the caudal tectum. We were particularly interested in comparing the relative density of axons in each tectal region with those previously determined in whole retinal regenerates (Stuermer,
$1988 a)$ as this would indicate whether more axons entered the inappropriate tectal half under these circumstances. The density and orientation of axons crossing a standard sample area was determined at six representative tectal sites in two animals for each datum point (short-term nasal and temporal, longterm nasal and temporal). These sites were located along lines joining the nucleus rotundus and the caudal ends of the slit and flattened tectum (Stuermer, 1988a) and comprised a medial and lateral region in rostral, middle and caudal tectum. At each site, the total number of all axons within a circular area of tectum $136 \mu \mathrm{m}$ in diameter was determined at a magnification of $\times 1000$.

The plot of relative axon density versus rostrocaudal tectal location (Fig. 1) clearly shows that temporal axonal density (solid lines) declined drastically from $70 \%$ in rostral tectum to $28 \%$ in midtectum and finally $2 \%$ in caudal tectum, at both stages examined, while nasal axons maintained an even density into the caudal tectum (rostral, $30 \%$; mid, $40 \%$; caudal, $30 \%$ ). This is very similar to whole retinal regenerates (Stuermer, 1988a). Therefore, both nasal and temporal axons exhibit an equally strong preference for their retinotopically appropriate tectal half whether or not the axons appropriate to the other tectal half are present.

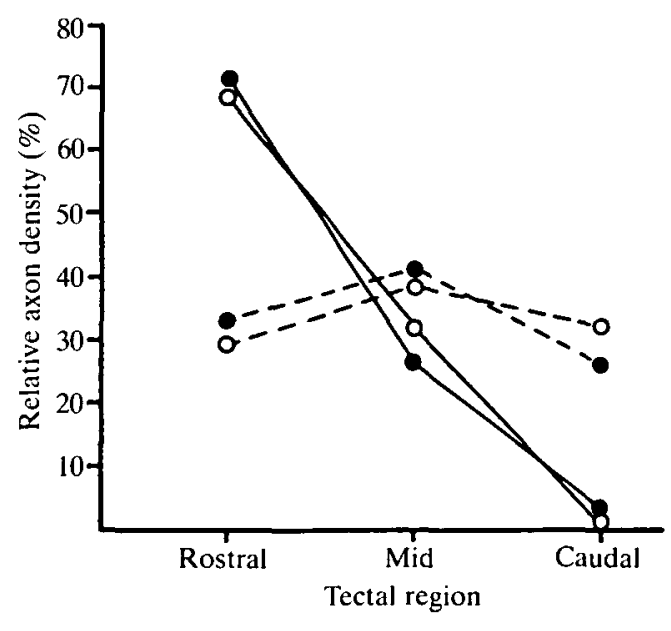

Fig. 1. Plot of relative axon density versus tectal region. See results for detailed explanation. The axonal densities within a standard sample area in each tectal region indicated were summed and then the density at each location expressed as a percentage thus providing a standardized measure of axonal distribution. Solid lines indicate temporal half retinal regenerates while broken lines indicate nasal half retinal regenerates. Open circles are 76-day regenerates while filled circles are 39-day regenerates. At both stages, the axonal density of temporal regenerates dropped dramatically in caudal tectum while that of nasal regenerates was similar throughout the tectum. 

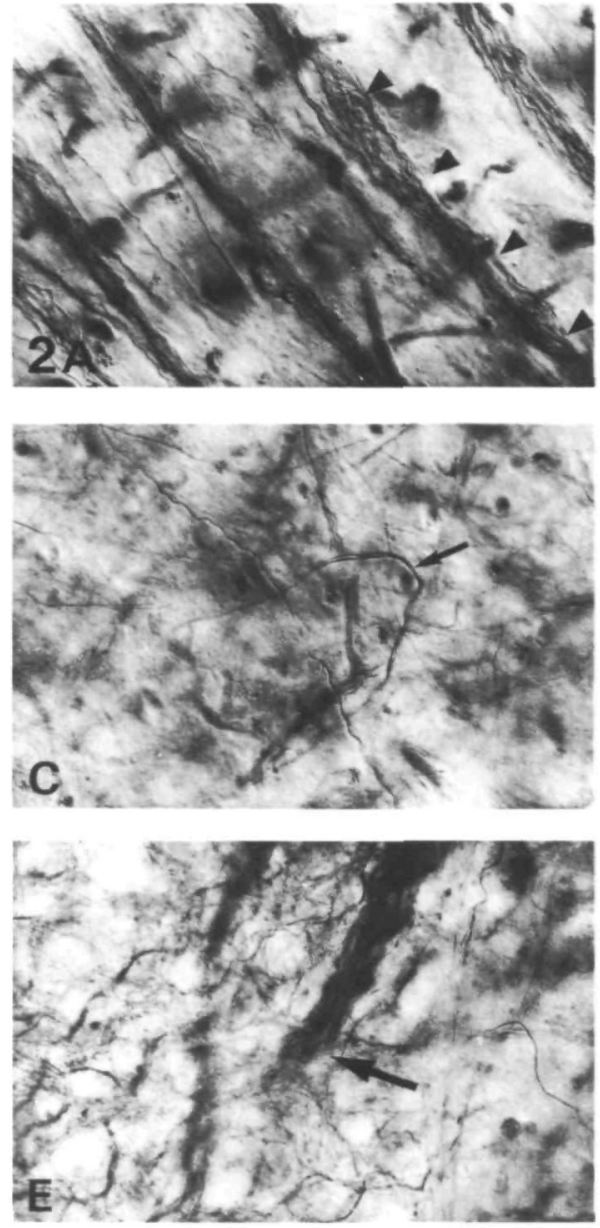
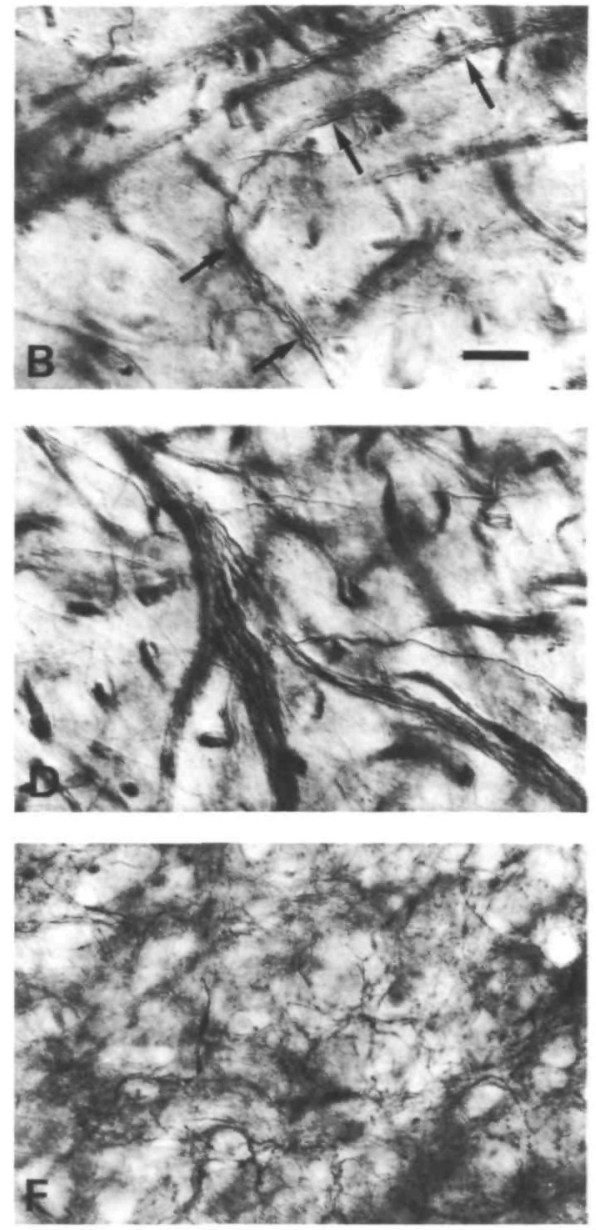

Fig. 2. Fascicle patterns.

(A) Fascicles (arrowheads) in the stratum opticum of a temporal half retinal regenerate. (B) A lateral fascicle bending sharply into a mediolateral orientation (arrows), temporal half retinal regenerate. (C) A small fascicle of temporal axons bending sharply in the synaptic layer of the midtectum.

(D) Large fascicles crossing the tectal equator in a temporal half retinal regenerate. (E) The rapid termination of a fascicle of temporal axons in a whole retinal regenerate (arrow). Note how the axons immediately disperse upon entering the synaptic layer. (F) The synaptic layer in the rostral tectum of a whole retinal regenerate where only the temporal axons were labelled. Note the even meshwork of axons and absence of fascicles.

Bar, $200 \mu \mathrm{m}$.

\section{Fascicle formation}

Both nasal and temporal axons formed fascicles within stratum opticum (Fig. 2A,B). Either temporal axons left the fascicles individually in rostral tectum or the fascicles themselves curved in a mediolateral orientation, in many cases crossing the tectal equator, and therefore did not enter caudal tectum (Fig. 2B). Groups of axons making similar loops were also found in the synaptic layer near the middle of the rostrocaudal extent of the tectum (Fig. 2C). Such pathways are rare or absent in the normal projection and were not described by Attardi \& Sperry (1963).

In whole retinal regenerates, axons were often found to exit from fascicles and cross the tectal equator in the deeper, synaptic layer apparently to correct for having entered via the incorrect brachium (Stuermer, 1988a). It was very evident in the current study that the temporal ingrowth tecta contained many large mediolaterally oriented fascicles crossing the rostral tectal equator in deeper layers (Fig. 2D). Such fascicles are occasionally seen in whole retinal regenerates but the frequency appeared much higher in the temporal half retinal regenerates. Many temporal ingrowth fascicles abruptly changed their orientation to mediolateral, entered the synaptic layer as an intact fascicle, which, in many cases, then crossed the tectal equator. In contrast, the majority of fascicles in whole retinal regenerates came to an abrupt end as they approached the tectal equator (Fig. 2E) and individual axons entered the meshwork of axons in the synaptic layer (Fig. 2F). Nasal axons formed a fascicle pattern similar to that of whole retinal regenerates and did not have an excessive degree of equatorial crossing. Those nasal axons that did enter the incorrect dorsal or ventral hemitectum crossed the equator as individuals rather than in fascicles.

To examine whether the degree of fasciculation in temporal half retinal regenerates was objectively greater than that of whole retinal regenerates, the fascicle pattern of an animal that had the nasal retina removed at the time of optic nerve cut was compared with that of an animal in which the whole retina had regenerated after nerve cut but only the temporal axons were labelled. It was clear that in the whole retinal regenerate, while some fascicles crossed the equator at the boundary between rostral and caudal tectum, the majority of fascicles dispersed into single fibres before reaching the equator (Fig. 3B). In contrast, the temporal half retinal regenerate contained large fascicles throughout the rostral tectum 
which were oriented mediolaterally and continued across the tectal equator (Fig. 3A). In peripheral tectum, the superficial fascicles of the whole and half retinal regenerates were similar although there was more fasciculation at deeper levels in the half retinal

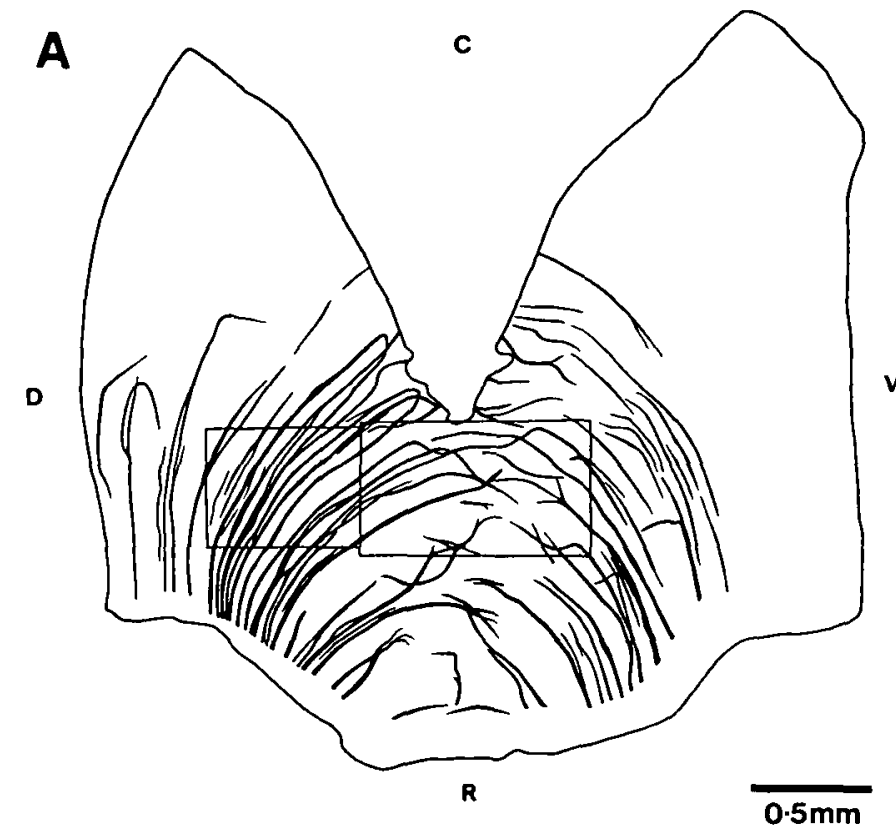

B

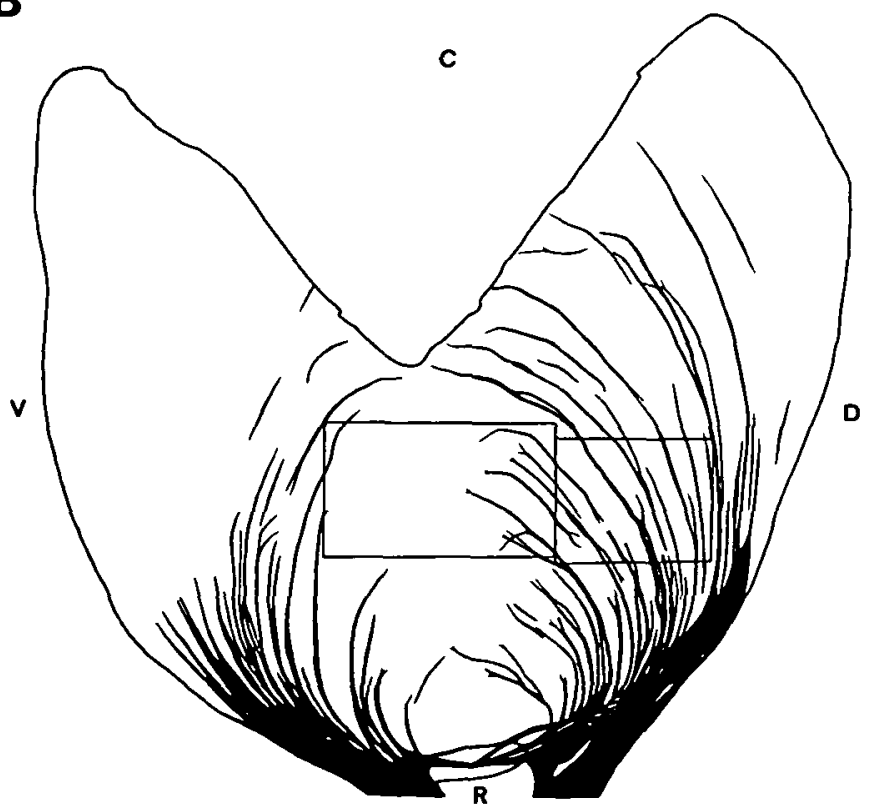

Fig. 3. Fascicular paths drawn at a magnification of $\times 160$ in a temporal retinal regenerate $(A)$, and a whole retinal regenerate $(B)$ in which only temporal axons were labelled. Both were labelled at 76 days after nerve cut. Note the increased crossing of the tectal equator in the half retinal regenerate and also the slightly greater spread of fascicles into caudal tectum in the whole retinal regenerate. $\mathrm{R}$, rostral; $\mathrm{C}$, caudal; $\mathrm{D}$, dorsal; $\mathrm{V}$, ventral. Boxes indicate the regions analysed in greater detail. regenerate. Drawings done at higher magnification $(\times 400$, oil immersion) also confirmed this pattern.

Thus, in the absence of nasal axons, temporal axons seemed to associate with one another to a greater degree within rostral tectum. Thus, many temporal axons crossed the tectal equator both individually and in fascicles. These crossings are found in all regenerates but rarely, if ever, in normals and are not therefore consistent with axons following normal pathways (Attardi \& Sperry, 1963). Also, fewer fascicles entered caudal tectum, possibly since no nasal axons were present to lead them astray. Nasal axons followed pathways similar to those of whole retinal regenerates crossing rostral tectum both in fascicles and via deep extrafascicular pathways.

\section{Single axon tracing}

Discrete bundles of axons from defined retinal locations were labelled in order to obtain information about the pathways taken by individual axons and their topographic appropriateness (six nasal removals, six temporal removals). Only the early stage of regeneration was examined in this manner since this was when the pathways were just being established in the tectum. In two nasal and two temporal half retinal ingrowth tecta, all the labelled axons were drawn. Other tecta were examined without drawing and found to confirm the growth patterns of the drawn tecta. At 35-40 days a large number of axons had growth cones at their leading edge and also on the tips of side branches if branching had occurred. This confirmed that the pathways were at an early stage of formation.

Compilation of all traced axons revealed a pattern similar to that of half retinal regenerates in which the whole nerve was labelled (Fig. 4A,B). Dorsotemporal axons had a tendency to be directed towards the midlateral tectum (Fig. 4A). Axons travelled towards this region in various ways. Some axons, particularly those far from the appropriate target region (often due to entering the incorrect brachium), continued in the fascicle layer without branching (Fig. 4A, small arrows). However, near the midtectum many axons were found whose paths were curved towards the appropriate termination region (Fig. 4A, large arrows). Those that had passed beyond the retinotopic region looped back (Fig. 4A, arrowheads), demonstrating a clear preference to search in rostral tectum. Within the rostral tectal half, axons outside the fascicles had branched, both close to their retinotopic region and at ectopic sites. In whole retinal regenerates where only the temporal axons were labelled, the majority of labelled axons branched in rostral tectum but a few axonal ramifications were also found in caudal tectum. In half retinal regenerates, fewer temporal axons invaded caudal tectum and no 
branching axons were observed in caudal tectum. Three typical temporal axons from Fig. 4A are shown separately in Fig. 4B for greater clarity (axons 1, 2 \&

A
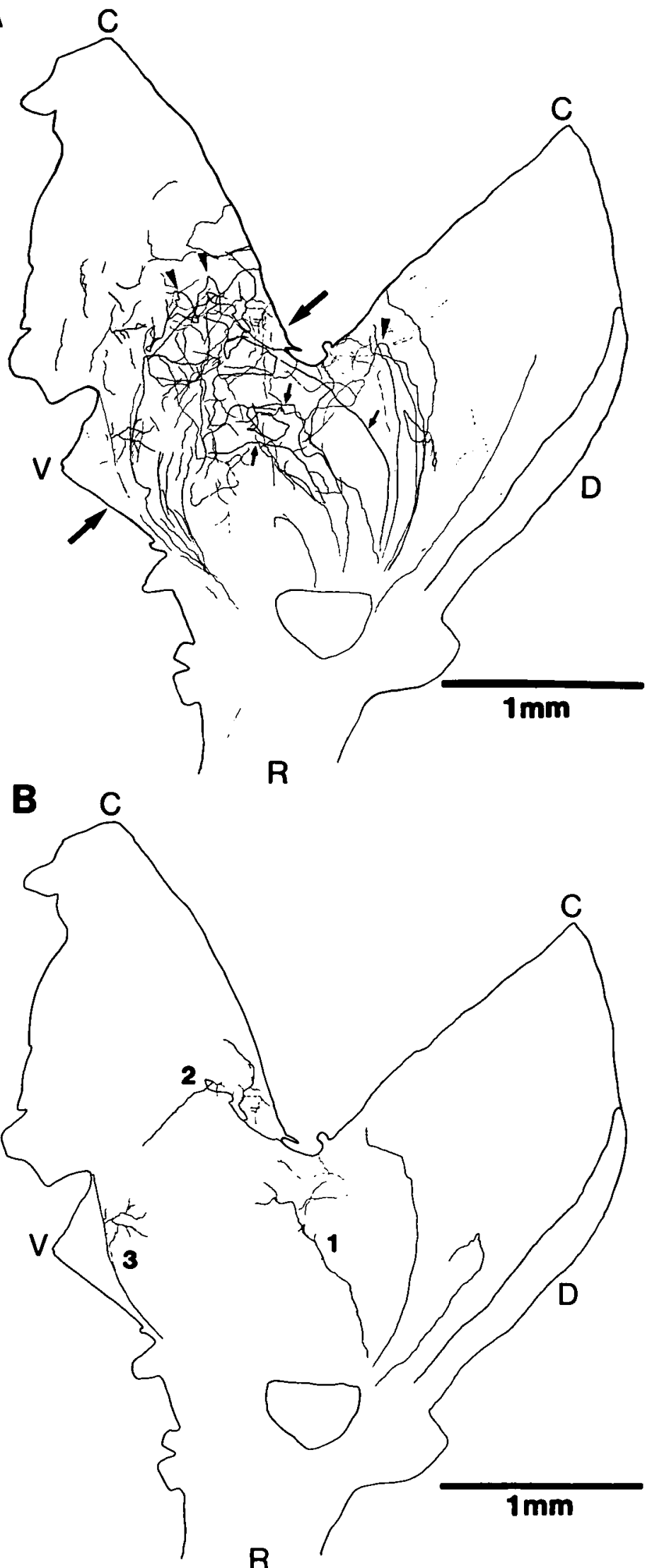

3 ). One of these axons apparently continued extending caudally, beyond the correct termination region, while maintaining an elaborate side branch directed towards the retinotopically appropriate region (Fig. 4B, axon 3). Possibly this axon would have eventually withdrawn the caudally directed process but we have no direct evidence of this. The other axons (Fig. 4B, axons $1 \& 2$ ) are clearly branching elaborately in the proximity of the correct region despite starting from relatively inappropriate tectal regions. Due to this type of course correction, the majority of temporal axons did not enter caudal tectum.

In the nasal ingrowth animals, it was evident that, although the axons had to grow through rostral tectum to reach their appropriate termination region, the majority of axons did not branch until entering caudal tectum (Fig. 5A,B). Only a few axons extended branches into rostral tectum (Fig. 5A, arrowheads). Fig. 5B shows the branching in caudal tectum more clearly and also examples of axons that cross rostral tectum without forming any elaborate processes. Those axons with growth cones at their tips within rostral tectum were usually directed towards the caudal tectum.

Longer term nasal and temporal half retinal regenerate tecta were examined for the presence of mature arbours. In each case, they were only found in the retinotopically appropriate tectal half. The morphology of axons branching in the inappropriate tectal half was always immature.

In summary, the tectal pathways of nasal and temporal axons were not greatly altered by the absence of their respective partners from the other half of the retina. The number of temporal axons invading the caudal tectum was no greater than in whole retinal regenerates even though the caudal tectum was vacant. On the contrary, many temporal axons made sharp course corrections to avoid the retinotopically inappropriate tectal half. Neither did nasal axons exhibit any increased tendency to enter and branch in the 'vacant' SGFS of the rostral tectal

Fig. 4. Single axon tracing - temporal axons. Thick lines indicate the tectal outline. The retinotopically appropriate termination region for the labelled axons is indicated by the large arrows. (A) Overall pattern. Most axons are directed towards and branch within the retinotopically appropriate region. Some axons reach this region by crossing the tectal equator because they entered the dorsal brachium (small arrows). Others turn sharply away from caudal tectum (large arrowheads) and some continue into caudal tectum. (B) Examples of widely branching axons $(1,2)$ and an axon (3) continuing to grow caudally despite an elaborate side branch directed towards the appropriate region. 
half, in order to innervate rostral tectum, but continued into caudal tectum before branching. The whole-nerve-labelled animals demonstrated a con- solidation of this pattern at later stages with no greater tendency for the later arriving axons to spread into the wrong tectal half.

A

B

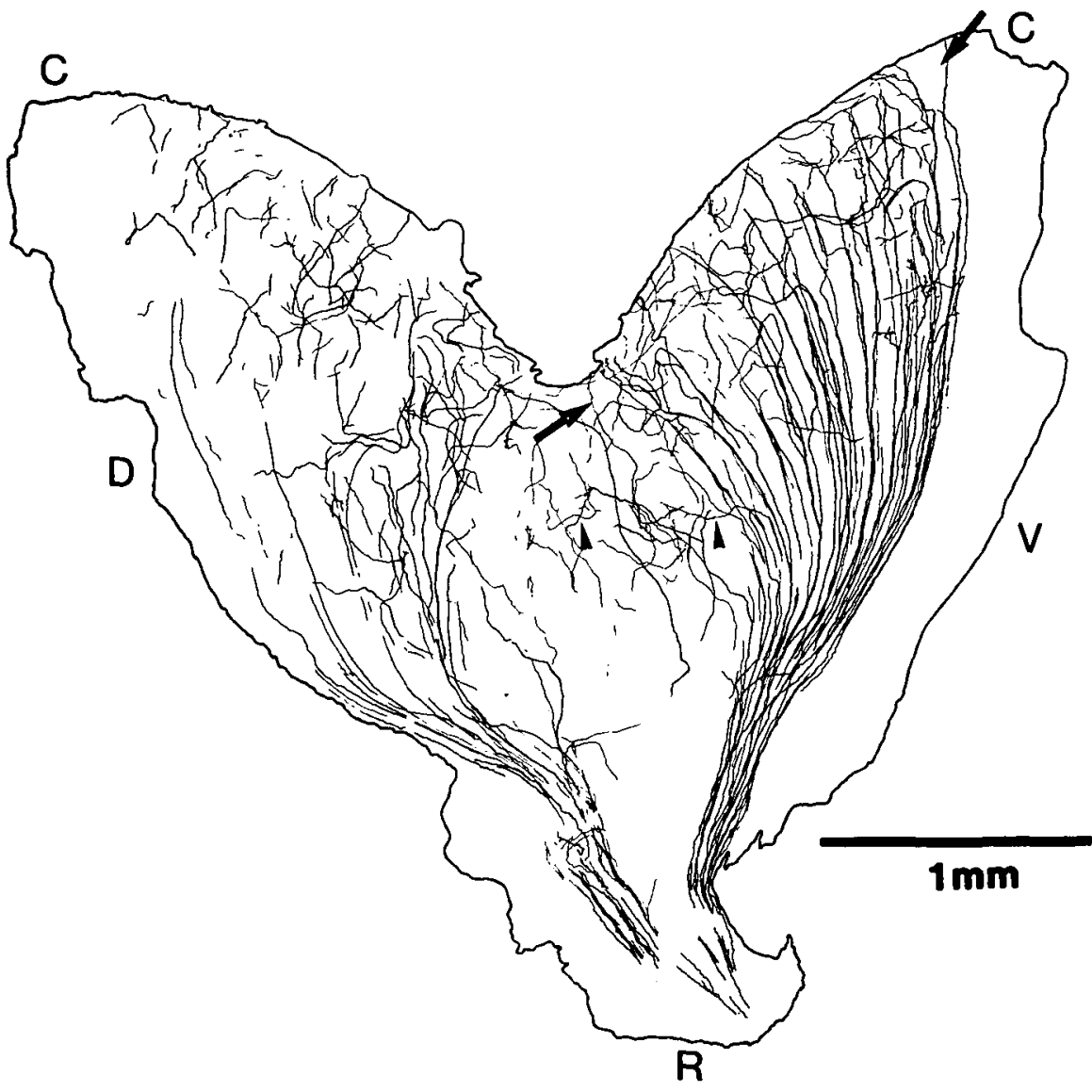

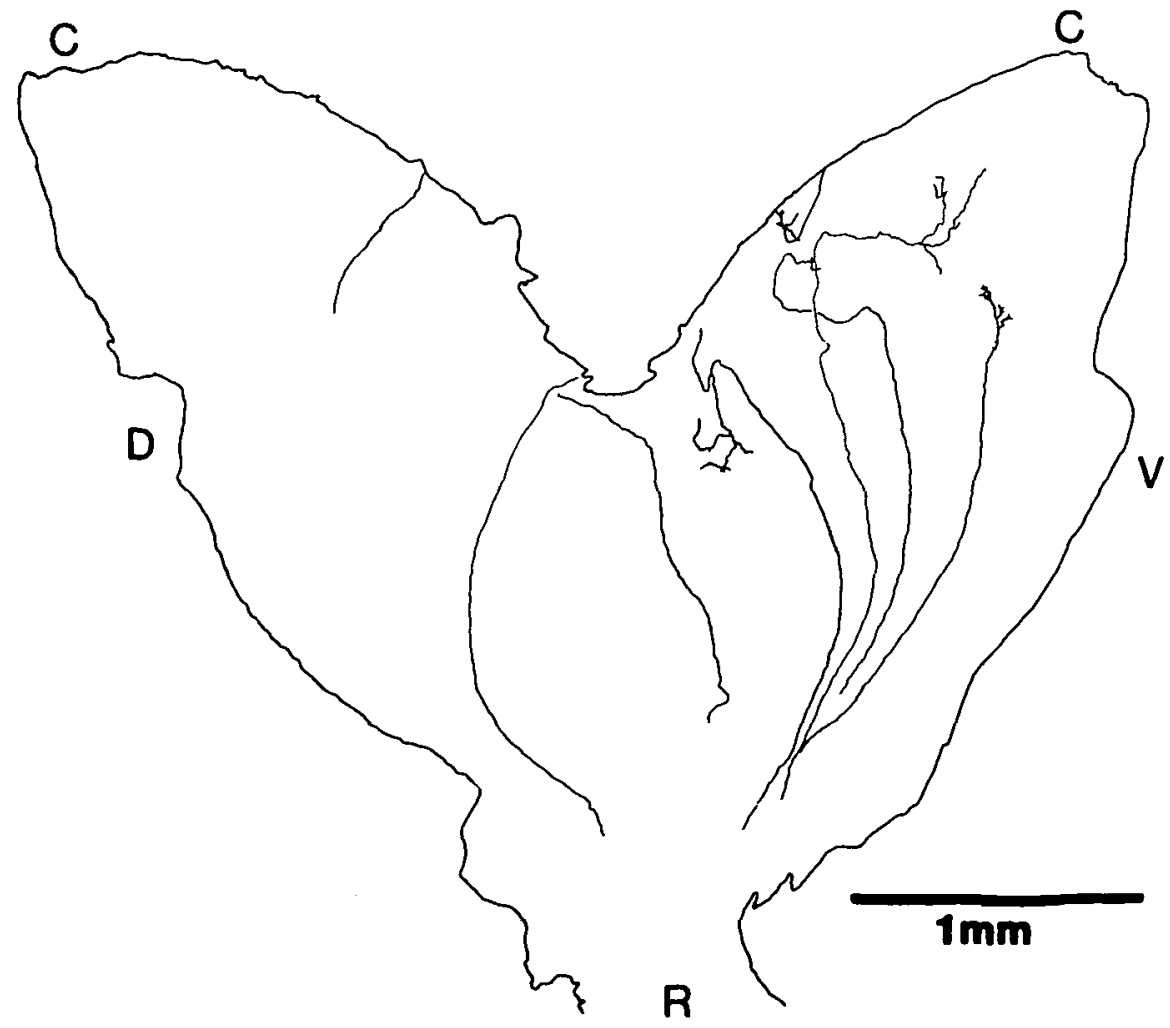

Fig. 5. Single axon tracing nasal axons. Conventions are as in Fig. 4. (A) Overall pattern. The majority of axons branch in the retinotopically appropriate region and traverse rostral tectum in simple fascicular paths. Some axons extend branches into rostral tectum (arrowheads). (B) Examples of nasal axons branching in caudal tectum. 


\section{Discussion}

The major conclusion of this study is that even when half of the retina is removed, a procedure that prevents certain interactions between axons and leaves a large area of tectum without its appropriate innervation, both nasal and temporal axons exhibit a preference to grow through their retinotopic tectal half. The semiquantitative methods used allow a direct comparison with similar analyses done on nasal and temporal axons in whole retinal regenerates (Stuermer, 1988a,b). Axons exhibit similar preferences during whole retinal regeneration even though, as in half retinal regenerates, they follow many inappropriate routes through the tectum. Interaxonal interactions cannot therefore be of major importance in this specificity since the degree of preference is similar in these two situations.

In whole retinal regeneration, a low proportion of temporal axons extend into the inappropriate caudal tectal half (Stuermer, 1986, 1988a,b). The present study has demonstrated that the proportion of these axons is no greater in the absence of axons appropriate to the caudal tectum. In fact, fewer temporal axons entered the caudal tectum via fascicles. This suggests that axons that enter the caudal tectum through fascicular pathways in whole retinal regenerates do so because they are following nasal axons. Similarly, nasal axons of half retinal regenerates were distributed as in whole retinal regenerates even though they had to pass through rostral tectum which lacked its appropriate innervation. Thus, denervated tectum appears not to be attractive to ingrowing axons unless it is of the appropriate retinotopic specificity.

Thus, with minor exceptions, the pattern of both nasal and temporal axonal innervation is the same whether they regenerate together or separately. Attardi \& Sperry (1963) reached similar conclusions, however, the limitations of the techniques available at the time led them to assume that regenerating axons follow their previous pathways. Numerous recent studies have shown that, on the contrary, regenerating axons take abnormal routes through the tectum but nevertheless relocate their retinotopic termination sites (Cook, 1983; Rankin \& Cook, 1986; Stuermer, 1986, 1988a,b; Stuermer \& Easter, 1984b). Analysis of these pathways shows that axons do not err randomly either in whole retinal regeneration (Stuermer, 1988a,b) or when large regions of the tectum are left without appropriate innervation (current study). Since the restriction of axons to their appropriate tectal half is not due to competitive interactions between the axons and since axonal pathways are directed to their correct termination regions despite considerable pathway disorganization, we favour the view that axons follow positional cues present in the tectal neuropil. Studies of retinotectal projection formation during development in Xenopus and zebra fish also found no indication of interaxonal competition affecting the initial arborization pattern (Holt, 1984; Stuermer, 1988c). Therefore, recognition of tectal positional cues by optic axons seems to play a major role in the initial topography of various systems.

In half retinal regeneration, it is not possible for the age-related order of normal fascicles (Cook \& Rankin, 1984; Easter \& Stuermer, 1984; Stuermer \& Easter, 1984a) to be reproduced and yet fascicle formation was very obvious in these animals, although crossing of the tectal equator was very frequent for temporal axons. In whole retinal regenerates, a fascicle pattern also forms which is very reminiscent of that in normals, however, the organization is of a much lower grade and fascicles sometimes cross the tectal equator (Cook, 1983; Stuermer $\&$ Easter, 1984b; Stuermer, 1986). Reformation of a fascicle pattern in this study is therefore further evidence that the tendency to fasciculate in stratum opticum is more related to the tectal environment than axonal interactions.

Temporal axons of half retinal regenerates tended to associate very closely and often remain in fascicles even after turning in a mediolateral direction. This increased fasciculation probably reflects several factors. With no nasal axons to lead temporal axons astray they may form more obvious mediolateral fascicles. Additionally, the absence of nasal axons intermingled with temporal axons in the fascicles may lead to a closer association which is maintained when the axons leave stratum opticum. Finally, there may be an inherent tendency for temporal axons to associate more closely which is expressed in these circumstances (Bonhoeffer \& Huf, 1985; Gaze \& Fawcett, 1983).

The majority of nasal axons in half retinal regenerates crossed rostral tectum within fascicles and only exited from the fascicles in caudal tectum, thus forming long fascicular paths in stratum opticum. The finding of Meyer (1987) that responses could be recorded from rostral tectum at 44-64 days in nasal half retinal regenerates is not readily explained by our anatomical results. No mature arbours were found in the rostral tectum of nasal half retinal regenerates at these stages in this study. Some axons did, however, form immature branches and it is possible that careful recording would detect these.

Optic axons certainly have the capacity for wideranging growth in the tectum as demonstrated by their ability to innervate a surgically isolated tectal island (Edwards et al. 1985), however, the majority 
remain restricted to their appropriate tectal half. Much of this restriction evidently occurred in the stratum opticum, with comparatively few cases of wide rostrocaudal growth in stratum opticum subsequently corrected by pathways in the synaptic layer. This suggests that some tectal positional information must be available in stratum opticum.

The nature of the positional information in the fascicular layer is uncertain. The ingrowing axons are unmyelinated and usually tightly clustered around one another (Murray, 1976; Murray \& Edwards, 1982; Wolburg \& Bouzehouane, 1986). The fascicles are located in the same position as the fascicles of the previous projection and sometimes contact glial cells or other elements of the tectal neuropil. Although a large part of the axonal environment is composed of other axons we have seen that elimination of a substantial number of them did not influence the distribution of the fascicles. It is therefore most likely that the axons are influenced by markers on other elements. Although most of the axons from the previous projection have degenerated by the time that the regenerating axons enter, it is possible that they are following the remnants of the previous projection, but in a rather nonspecific way. It has also been suggested that the previous projection leaves 'markers' imprinted on the tectum which allows organization of the regenerating axons (Schmidt, 1978). The role of the previous projection is currently uncertain. Some studies suggest that it plays a major role and that long-term denervation prior to regeneration allows immediate expansion of a half retinal projection (Schmidt, 1978). Other studies, however, show minimal effects of long-term denervation (Meyer, 1978, 1984, 1987; Busse \& Stuermer, 1987). One study in particular shows that the fascicular pathways in long-term denervated tecta are similar to those after simple regeneration suggesting that degenerating debris from the previous projection is not necessary for these pathways to form (Busse \& Stuermer, 1987). Furthermore, these axons terminate at their retinotopically appropriate sites, substantiating the view that long-lasting markers inherent to the tectum guide the growing axons.

At early stages of regeneration, the axons ramified widely in the synaptic layer of the tectum, having many fine branches with growth cones at their tips. Single axon tracing showed that, as in whole retinal regenerates, these fine arbours were not always located in the appropriate tectal region. At later stages, only few immature branches were located in the inappropriate tectal half and mature arbour formation was confined to the appropriate tectal half. This sequence of events is consistent with the early branching being a strategy for more accurately sensing tectal cues and thereby finding a pathway to the appropriate termination site (Fujisawa et al. 1982; Stuermer, 1987b) rather than simply spreading to cover as much denervated tectum as possible prior to selecting appropriate branches. The axons that enter the inappropriate tectal half in half retinal regeneration have no axons with which to compete and therefore competition between axons cannot be a factor in their growth out of the incorrect region.

MFH would like to thank Prof. $\mathrm{H}$. Wässle for provision of facilities and J. Sapp for typing the manuscript.

\section{References}

Attardi, D. G. \& Sperry, R. W. (1963). Preferential selection of central pathways by regenerating optic fibers. Expl Neurol. 7, 46-64.

Bonhoeffer, F. \& HuF, J. (1985). Position-dependent properties of retinal axons and their growth cones. Nature, Lond. 315, 409-410.

Busse, U. \& Stuermer, C. A. O. (1987). Navigation and target recognition of regenerating retinal axons in longterm denervated tecta in goldfish. Neurosci. Abstr. 13, 1418

Cook, J. E. (1983). Tectal paths of regenerated optic axons in goldfish: evidence from retrograde labelling with horseradish peroxidase. Expl Brain Res. 51, 433-442.

Cook, J. E. \& Rankin, E. C. C. (1984). Use of a lectin-peroxidase conjugate (WGA-HRP) to assess the retinotopic precision of goldfish optic terminals. Neurosci. Lett. 48, 61-66.

Easter, S. S., JR \& Stuermer, C. A. O. (1984). An evaluation of the hypothesis of shifting terminals in goldfish optic tectum. J. Neurosci. 4, 1052-1063.

Edwards, M. A., Sharma, S. C. \& Murray, M. (1985). Selective retinal reinnervation of a surgically created tectal island in goldfish. I. Light microscopic analysis. J. comp. Neurol. 232, 372-385.

Fujisawa, H., Tani, N., Watanabe, K. \& Ibata, Y. (1982). Branching of regenerating retinal axons and preferential selection of appropriate branches for specific neuronal connections in the newt. Devl Biol. 90, 43-57.

Gaze, R. M. \& Fawcett, J. W. (1983). Pathways of Xenopus optic fibres regenerating from normal and compound eyes under various conditions. J. Embryol. exp. Morph. 73, 17-38.

Holt, C. E. (1984). Does timing of axon outgrowth influence initial retinotectal topography in Xenopus? J. Neurosci. 4, 1130-1152.

Humphrey, M. F. \& Stuermer, C. A. O. (1987). Tectal pathways of regenerating goldfish optic axons after half-nasal or half-temporal retinal removal. Neurosci. Abstr. 13, 1419.

Meyer, R. L. (1978). Deflection of selected optic fibers into a denervated tectum in goldfish. Brain Res. 155 , 213-227. 
Meyer, R. L. (1979). Retinotectal projection in goldfish to an inappropriate region with a reversal in polarity. Science 205, 819-821.

MeYer, R. L. (1980). Mapping the normal and regenerating retinotectal projection of goldfish with autoradiographic methods. J. comp. Neurol. 189, 273-289.

MeYer, R. L. (1984). Target selection by surgically misdirected optic fibers in the tectum of goldfish. $J$. Neurosci. 4, 234-250.

Meyer, R. L. (1987). Tests for relabelling the goldfish tectum by optic fibers. Devl Brain Res. 31, 312-318.

Meyer, R. L., Sakurai, K. \& Schauwecker, E. (1985). Topography of regenerating optic fibers in goldfish traced with local wheat germ injections into retina: Evidence for discontinuous microtopography in the retinotectal projection. J. comp. Neurol. 239, 27-43.

Murray, M. (1976). Regeneration of retinal axons into the goldfish optic tectum. J. comp. Neurol. 239, 27-43.

Murray, M. \& Edwards, M. A. (1982). A quantitative study of the reinnervation of the goldfish optic tectum following optic nerve crush. J. comp. Neurol. 209, 363-373.

Rankin, E. C. C. \& Cook, J. E. (1986). Topographic refinement of the regenerating retinotectal projection of the goldfish in standard laboratory conditions: a quantitative WGA-HRP study. Expl Brain Res. 63, 409-420.

Schmidt, J. T. (1978). Retinal fibers alter tectal positional markers during the expansion of the half retinal projection in goldfish. J. comp. Neurol. 177, 279-300.

Schmidt, J. T. \& Edwards, D. L. (1983). Activity sharpens the map during the regeneration of the retinotectal projection in goldfish. Brain Res. 269, 29-39.
Sharma, S. C. \& Tung, Y. L. (1979). Interactions between nasal and temporal hemiretinal fibers in adult goldfish tectum. Neurosci. 4, 113-119.

Stuermer, C. A. O. (1981). Modified retinotectal projection in goldfish: a consequence of the position of retinal lesions. In Lesion-Induced Neuronal Plasticity in Sensorimotor Systems (ed. H. Flohr \& W. Precht). Berlin, Heidelberg: Springer-Verlag.

Stuermer, C. A. O. (1984). Rules for retinotectal terminal arborizations in the goldfish optic tectum: A whole-mount study. J. comp. Neurol. 229, 214-232.

Stuermer, C. A. O. (1986). Pathways of regenerated retinotectal axons in goldfish. 1 . Optic nerve, tract and tectal fascicle layer. J. Embryol. exp. Morph. 93, 1-28.

StUERmer, C. A. O. (1988a). The trajectories of regenerating retinal axons in the goldfish tectum. I. A comparison of normal and regenerated axons at late regeneration stages. J. comp. Neurol. 267, 55-68.

Stuermer, C. A. O. (1988 $b)$. The trajectories of regenerating retinal axons in the goldfish tectum. II. Exploratory branches and growth cones on axons at early regeneration stages. J. comp. Neurol. 267, 69-91.

Stuermer, C. A. O. (1988c). Retinotopic organization of the developing retinotectal projection in zebra fish embryos. J. Neurosci. (in press).

Stuermer, C. A. O. \& Easter, S. S., Jr (1984a). Rules of order in the retinotectal fascicles of goldfish. $J$. Neurosci. 4, 1045-1051.

Stuermer, C. A. O. \& Easter, S. S., Jr (1984b). A comparison of the normal and regenerated retinotectal pathways of goldfish. J. comp. Neurol. 223, 57-76.

Wolburg, H. \& Bouzehouane, U. (1986). Comparison of the glial investment of normal and regenerating fiber bundles in the optic nerve and optic tectum of the goldfish and crucian carp. Cell Tissue Res. 244, 187-192.

(Accepted 17 November 1987) 